\section{SAT0431 THE ACR-EULAR CLASSIFICATION CRITERIA IN PRIMARY SJOGREN'S SYNDROME: THE CONTRIBUTING ROLE OF ULTRASOUND}

E. Mossel ${ }^{1}$, J.F. van Nimwegen ${ }^{1}$, M.S. van Ginkel ${ }^{1}$, K. Delli ${ }^{2}$, A.J. Stel ${ }^{1}$, F. G. Kroese ${ }^{1}$, F.K. Spijkervet ${ }^{2}$, A. Vissink ${ }^{2}$, S. Arends ${ }^{1}$, H. Bootsma ${ }^{1} .{ }^{1}$ Rheumatology and Clinical Immunology; ${ }^{2}$ Oral and Maxillofacial Surgery, University Medical Center Groningen, Groningen, Netherlands

Background: Salivary gland ultrasound (SGUS) is cheap, non-invasive and easy to perform in an outpatient setting. The ACR-EULAR criteria were recently developed to reach international consensus regarding the classification of primary Sjögren's syndrome (pSS), but SGUS is not yet included as a classification item. Objectives: To assess the performance of the ACR-EULAR criteria when salivary gland ultrasound (SGUS) replaces current items, in a large cohort of patients clinically suspected or diagnosed with pSS in daily clinical practice.

Methods: Included were all consecutive outpatients who underwent SGUS between October 2014 and, July 2017 and had a complete data-set on all ACREULAR items. Classification according to the criteria was determined separately in patients who were subjected to a labial or parotid gland biopsy. For SGUS, the average score for hypoechogenic areas in the parotid and submandibular glands on one side was applied (range $0-3)^{1}$. The optimal cut-off value for our SGUS score was determined using ROC analysis. Clinical diagnosis by the treating physician was used as gold standard. Area under the curve (AUC), absolute agreement, sensitivity and specificity of the original and adjusted ACR-EULAR criteria sets were determined.

Results: Of the 363 consecutive patients, 243 patients had a complete data-set, of whom 147 patients were diagnosed with pSS. Accuracy of SGUS to predict clinical diagnosis was good, with an AUC of 0.860 and optimal cut-off value of $\geq 1.5$. When applying a weight of 1 point for a positive SGUS, the cut-off value of the ACR-EULAR criteria to discriminate between pSS and non-pSS remained 4 , irrespective of the type of biopsy used.

In patients who underwent a labial gland biopsy ( $n=124)$, the original ACR-EULAR criteria showed an AUC of 0.965 (figure $1 \mathrm{~A}$ ). Absolute agreement with clinical diagnosis was $94.4 \%$, sensitivity was $95.9 \%$ and specificity was $92.2 \%$. When SGUS replaced the labial gland biopsy, absolute agreement was $87.9 \%$, sensitivity was $82.2 \%$ and specificity was $96.1 \%$. When SGUS replaced anti-SSA antibody status, absolute agreement was $89.5 \%$, sensitivity was $86.3 \%$ and specificity was $94.1 \%$. When SGUS replaced the ocular staining score (OSS), Schirmer's test or unstimulated whole saliva flow (UWS), absolute agreement varied between $89.5 \%-93.5 \%$, sensitivity varied between $90.4 \%-95.9 \%$ and specificity varied between $88.2 \%-92.2 \%$. In patients who underwent a parotid gland biopsy ( $n=198)$, similar results were found (figure 1B).
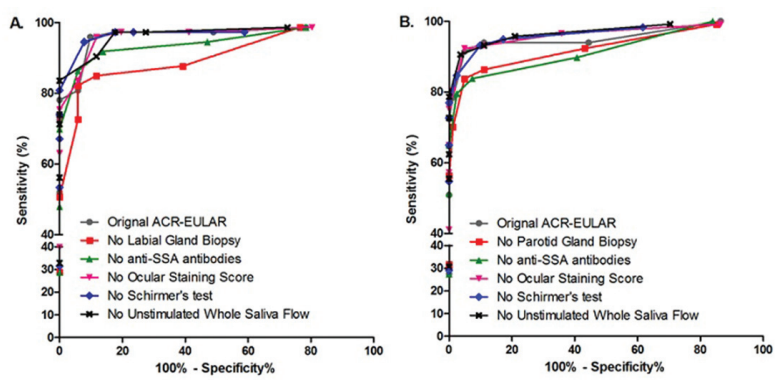

Abstract SAT0431 - Figure 1. Ultrasound replacing current ACR-EULAR items in patients who underwent a labial gland biopsy $(A)$ or a parotid gland biopsy (B).

Conclusions: SGUS cannot be used as a replacement for salivary gland biopsy or anti-SSA antibody status in the ACR-EULAR criteria because of a substantial reduction in sensitivity. For diagnostic purposes, a high sensitivity is preferred over a high specificity.

Replacement of the OSS, Schirmer's test or UWS by SGUS only resulted in negligible changes in accuracy of the ACR-EULAR criteria. With SGUS being able to replace one of these function tests, clinicians are offered more options that could lead to fulfilment of the ACR-EULAR criteria.
REFERENCE:

[1] Mossel, et al. Ann Rheum Dis 2017: Published online first at December 12.

Disclosure of Interest: None declared

DOI: 10.1136/annrheumdis-2018-eular.6779

\section{SAT0432 MEMBRANOUS VERSUS PROLIFERATIVE LUPUS NEPHRITIS: TWO DIFFERENT DISEASES?}

F. Farinha ${ }^{1}$, B.S. Bernstein ${ }^{1}$, R. Pepper ${ }^{2}$, D.A. Isenberg ${ }^{1}$, A. Rahman ${ }^{1} .{ }^{1}$ Centre for Rheumatology, ${ }^{2}$ Centre for Nephrology - Royal Free Campus, UCL, London, UK

Background: Lupus nephritis (LN) is currently classified according to the 2003 International Society of Nephrology/Renal pathology Society (ISN/RPS) classification system, which is based on histology. Most patients have proliferative lupus nephritis (PLN), which has been the most studied type of LN. Membranous lupus nephritis (MLN) is less frequent, accounting for $10 \%-20 \%$ of the cases. In some patients there is a combination of the two types.

Objectives: To compare MLN and PLN with respect to demographic, clinical and laboratory characteristics.

Methods: Single-centre retrospective observational study. All patients with biopsy-proven proliferative (class III and IV), membranous (class V) and mixed (class III or IV+V) LN (according to the 2003 ISN/RPS classification), followed at UCLH Rheumatology department from 1975 to 2017, were included. Individual clinical files were reviewed to obtain demographic, clinical, laboratory and pathological data. We also recorded data on treatment with corticosteroids, immunosuppressants and antimalarials. We compared groups using Pearson's chisquared test for qualitative variables and Mann-Whitney test for quantitative variables. Renal survival was analysed through the Kaplan-Meier method. Significance level was defined at 0.05 .

Results: 187 patients were included (table 1). Age at diagnosis was not significantly different between groups $(p=0.474)$. The groups differ regarding ethnicity higher proportion of Caucasians with PLN versus higher proportion of Afro-Caribbeans with MLN. Patients with MLN present with higher $\mathrm{C} 3$ levels and significantly lower anti-dsDNA levels than the ones with proliferative changes. Thirty-four patients with PLN, 3 with MLN and 2 with mixed nephritis, progressed to endstage renal disease. Cumulative renal survival rates at $5,10,15$ and 20 years were $91,81,75 \%$ and $66 \%$ for PLN and $100,97,92 \%$ and $84 \%$ for MLN, respectively (Image 1).

Abstract SAT0432 - Table 1. Comparison between the three groups of patients

\begin{tabular}{|c|c|c|c|c|}
\hline & Class III and IV & Class V & $\mathrm{III}+\mathrm{V}$ or IV+V & $\mathrm{p}$ \\
\hline Total, N & 135 & 38 & 14 & \\
\hline $\mathbf{F}, \mathrm{N}(\%)$ & $123(91)$ & $33(87)$ & $11(79)$ & 0.303 \\
\hline M, N (\%) & $12(9)$ & $5(13)$ & $3(21)$ & \\
\hline Caucasian, N (\%) & $67(50)$ & $12(32)$ & $3(21)$ & 0.044 \\
\hline Afro-Caribbean, N (\%) & $35(26)$ & $18(47)$ & $6(43)$ & \\
\hline Asian, $\mathrm{N}(\%)$ & $33(24)$ & $8(21)$ & $5(36)$ & \\
\hline $\begin{array}{l}\text { uPCR at LN diagnosis, median; } \\
\text { IQR }\end{array}$ & $261.5 ; 372$ & $254.0 ; 276$ & $143.0 ; 195$ & 0.663 \\
\hline $\begin{array}{l}\text { Creatinine at LN diagnosis, } \\
\text { median; IQR }\end{array}$ & $73.5 ; 40$ & $54.5 ; 17$ & $73 ; 58$ & 0.106 \\
\hline $\begin{array}{l}\text { Albumin at LN diagnosis, median; } \\
\text { IQR }\end{array}$ & $32.5 ; 13$ & $31 ; 9$ & $35 ; 4$ & 0.624 \\
\hline C3 at LN diagnosis, median; IQR & $0.61 ; 0.34$ & $0.81 ; 0.57$ & $0.64 ; 0.32$ & 0.002 \\
\hline $\begin{array}{l}\text { Anti-dsDNA at LN diagnosis, } \\
\text { median; IQR }\end{array}$ & $863.0 ; 1616.75$ & $80 ; 149.5$ & $296 ; 242$ & 0.000 \\
\hline Ever Low C3, N (\%) & $107(80)$ & $35(92)$ & $11(79)$ & 0.203 \\
\hline Ever anti-dsDNA positive, $\mathrm{N}(\%)$ & $111(83)$ & $32(84)$ & $12(86)$ & 0.950 \\
\hline Ever anti-Sm positive, $\mathrm{N}(\%)$ & $25(19)$ & $16(42)$ & $6(43)$ & 0.004 \\
\hline Ever anti-RNP positive, $\mathrm{N}(\%)$ & $42(31)$ & $19(50)$ & $8(57)$ & 0.030 \\
\hline Ever anti-Ro positive, $\mathrm{N}(\%)$ & $54(40)$ & $16(42)$ & $10(71)$ & 0.081 \\
\hline Ever anti-La positive, $\mathrm{N}(\%)$ & $21(16)$ & $3(8)$ & $4(29)$ & 0.168 \\
\hline Use of antimalarials, $\mathrm{N}(\%)$ & $82(66)$ & $27(73)$ & $9(69)$ & 0.732 \\
\hline $\begin{array}{l}\text { Use of immunosuppressants, N } \\
(\%)\end{array}$ & $121(95)$ & $35(95)$ & $14(100)$ & 0.669 \\
\hline Use of corticosteroids, $\mathrm{N}(\%)$ & $125(97)$ & $36(95)$ & $13(93)$ & 0.668 \\
\hline
\end{tabular}

F: females; M: males; uPCR: urinary protein-creatinine ratio; LN: Lupus nephritis; FU: followup 